\title{
NEAR-IR PHOTOMETRIC STUDY OF THE FU ORIONIS OBJECT HBC 722
}

\author{
Hyun-Il Sung ${ }^{1}$, Won-Kee Park ${ }^{1}$, Yuna Yang ${ }^{2}$, Sang-Gak LeE ${ }^{2}$, Tae Seog Yoon ${ }^{3}$, Jeong-Eun LeE $^{4}$, \\ Wonseok Kang ${ }^{5}$, Keun-Hong Park ${ }^{2}$, Dong-Hwan Cho ${ }^{3}$, And Sunkyung Park ${ }^{4}$ \\ ${ }^{1}$ Korea Astronomy and Space Science Institute, Daejeon 305-348, Korea \\ E-mail : hisung@kasi.re.kr \\ 2 Department of Physics and Astronomy, Seoul National University, Seoul 151-742, Korea \\ ${ }^{3}$ Department of Astronomy and Atmospheric Sciences, Kyungpook National University, Daegu 702-701, Korea \\ 4 School of Space Research, Kyung Hee University, Yongin, Gyeonggi 446-701, Korea \\ 5 National Youth Space Center, Goheung, Jeollanamdo 548-951, Korea \\ (Received October 22, 2013; Accepted November 29, 2013)
}

\begin{abstract}
We present near-infrared light curves of HBC 722 after its the September 2010 outburst. We have been monitoring its near-infrared light curves since November 2010 with Korean Astronomy and Space Science Institute Infrared Camera System (KASINICS). HBC 722 exhibits large changes in optical and near-infrared brightness since its outburst. The $J, H$, and $K_{s}$ light curves over about 2.5 years show that in all observed bands HBC 722 progressively became fainter until around April 2011, down to $J \sim 10.7$, $H \sim 9.9, K_{s} \sim 9.3$, but it is getting brighter again. Large scatter in the obtained light curve prevents us from finding whether there is any short timescale variation as reported in other optical observations. The near-infrared color of HBC 722 is becoming bluer since its outburst. The pre-outburst Spectral Energy Distribution (SED) of HBC 722 is consistent with that of a slightly reddened Class II YSO with the exception of the extraordinary IR-excess in the far-infrared region.
\end{abstract}

Key words : stars: pre-main sequence — infrared: stars — stars: individual: HBC 722

\section{INTRODUCTION}

FU Orionis objects (FUors) are low mass pre-main sequence variables that show large outburst in optical light $(\Delta \mathrm{V}=4 \sim 6 \mathrm{mag})$ and remain bright for decades (Herbig 1966, 1977; Kolotilov \& Petrov 1983, 1985). Most of them are heavily extincted and have large infrared IR emission excesses (Weintraub et al. 1991). Detection of far-infrared emission from FUors reveals that they are still experiencing infall from the protostellar envelope. Infrared observations are required to understand the physical state of FUors in the early stellar evolution phase. Their episodic accretion bursts and luminosity flares are interesting phenomena in young stellar objects (YSOs) evolution, as well as in more general star formations studies. FUors are rare because the outburst cannot last long. Outbursts are thought to be short-lived phenomena $\left(\sim 10^{2} y r\right)$. Thus, it is hard to study the early state of FUors.

An enhanced accretion up to $10^{-4} M_{\odot} / y r$ and $\mathrm{P}$ Cygni line profiles in its spectrum are typical features of FUors. YSO outbursts are very interesting events because of their potential importance to the broader star and planet formation process. Low-mass stars could accrete up to half of their final mass during FUors outbursts (Hartmann \& Kenyon 1996), and their strong winds and outflows are likely to have a significant im-

Corresponding Author: H.-I. Sung pact on the surrounding interstellar medium (Croswell et al. 1987).

Only 20 FUors have been observed after the first observations and there were no outburst during several decades. HBC 722 (also known as V2493 Cyg, LkHa 188-G4, and PTF 10qpf) is a FU Orionis type young stellar object which showed an outburst on September 2010 (Semkov \& Peneva 2010; Miller et al. 2011). This new FUors was ideal to study the physical state of the protostellar envelope right after the outburst. The optical photometric variability was analyzes, and the large amplitude outburst and re-brightenig were clearly observed (Semkov et al. 2012; Green et al. 2011). Lee et al. (2011) presented the high resolution optical spectroscopic evolution of HBC 722 which showed the early stages of a FUors outburst. In this paper we present the near-infrared light curve of HBC 722 after its outburst. Using archival data we characterize its envelope via the spectral energy distribution and the near-infrared color-color diagram.

\section{OBSERVATION AND PHOTOMETRY}

\subsection{Observation}

The near-infrared observation of HBC 722 has been carried out with KASINICS (Moon et al. 2008) attached to the Cassegrain focus of the $1.8 \mathrm{~m}$ telescope at Bohyunsan Optical Astronomical Observa- 
Table 1.

KASINICS Photometric observations of HBC 722

\begin{tabular}{ccccccc}
\hline \hline Date & $\begin{array}{c}\text { Date } \\
(J D-2450000)\end{array}$ & $\begin{array}{c}\text { Seeing } \\
\left(^{\prime \prime}\right)\end{array}$ & $\begin{array}{c}\text { Exp. Time } \\
(\text { hour })\end{array}$ & $\begin{array}{c}J \\
(\mathrm{mag})\end{array}$ & $\begin{array}{c}H \\
(\mathrm{mag})\end{array}$ & $\begin{array}{c}K_{s} \\
(\mathrm{mag})\end{array}$ \\
\hline $2010-11-19$ & 5519.91 & 1.4 & 0.6 & 10.18 & 9.27 & 8.82 \\
$2010-11-24$ & 5524.93 & 2.5 & 2.5 & 10.27 & 9.33 & 8.83 \\
$2010-11-25$ & 5525.94 & 2.4 & 2.4 & 10.21 & 9.37 & 8.81 \\
$2011-03-22$ & 5643.36 & 3.3 & 1.1 & 10.71 & 9.70 & 9.04 \\
$2011-03-25$ & 5646.36 & 2.8 & 1.1 & 10.66 & 9.63 & 9.08 \\
$2011-04-03$ & 5655.34 & 2.3 & 1.3 & 10.58 & 9.68 & 9.13 \\
$2011-04-28$ & 5680.21 & 2.2 & 1.0 & 10.60 & 9.69 & 9.11 \\
$2011-10-11$ & 5846.06 & 1.7 & 1.3 & 10.41 & 9.40 & 8.87 \\
$2011-10-13$ & 5848.01 & 1.7 & 1.0 & 10.41 & 9.38 & 8.88 \\
$2011-10-18$ & 5853.14 & 1.6 & 0.8 & 10.45 & 9.49 & 8.96 \\
$2011-11-20$ & 5885.96 & 3.1 & 3.0 & 10.38 & 9.38 & 8.86 \\
$2011-11-21$ & 5886.97 & 2.0 & 3.8 & 10.30 & 9.32 & 8.84 \\
$2012-01-14$ & 5940.89 & 2.2 & 0.3 & 10.05 & 9.14 & 8.61 \\
$2012-03-13$ & 6000.34 & 2.3 & 1.1 & 10.03 & 9.07 & 8.60 \\
$2012-05-08$ & 6056.25 & 1.8 & 0.5 & 9.85 & 9.00 & 8.54 \\
$2012-05-09$ & 6057.23 & 2.4 & 3.2 & 9.83 & 8.96 & 8.38 \\
$2012-05-18$ & 6066.24 & 2.1 & 3.1 & 9.89 & 8.98 & 8.48 \\
$2012-05-20$ & 6068.22 & 2.0 & 4.2 & 9.88 & 8.96 & 8.47 \\
$2012-09-19$ & 6190.03 & 1.9 & 3.3 & 9.68 & 8.74 & 8.32 \\
$2012-09-20$ & 6191.07 & 1.7 & 2.7 & 9.70 & 8.77 & 8.37 \\
$2012-10-23$ & 6224.00 & 1.8 & 5.2 & 9.63 & 8.74 & 8.28 \\
$2012-10-25$ & 6226.03 & 1.5 & 6.8 & 9.65 & 8.68 & 8.18 \\
$2013-05-30$ & 6443.23 & 1.9 & 3.4 & 9.36 & 8.46 & 7.95 \\
$2013-06-04$ & 6448.23 & 1.5 & 2.0 & 9.31 & 8.41 & 7.90 \\
\hline
\end{tabular}

$\dagger$ Mean value for all the individual $K_{s}$ images from the night.

tory $(\mathrm{BOAO})$ in Korea. The first observation started in November 2010, about two months after the outburst peak occurred. Since then, we kept monitoring its brightness variation with the same camera for 24 nights during over 2.5 years. Table 1 shows a brief summary of our HBC 722 monitoring activity.

HBC 722 was observed in $J, H$, and $K_{s}$ bands. The exposure time for an individual image was limited to less than 20 seconds, because the background sky level in the near-infrared band is high and variable, which would decrease the photometric confidence in longer exposures. We also considered the detector linearity and saturation level in order to determine the exposure time. The detector of KASINICS undertakes the linearity between 4000 and 16000 ADU within 1\% accuracy and is saturated over 22500 ADU (Moon et al. 2008). The ADU value of all the images is in this linearity-guaranteed range.

KASINICS has a $3.3^{\prime} \times 3.3^{\prime}$ field of view, and since HBC 722 is located in a crowded star forming region, we made an observation of an additional adjacent field with few stars, to estimate the background sky level between the observation of the HBC 722 field. Fig. 1 shows an example of a KASINICS image for HBC 722. The target, comparison and check stars are marked in the figure.

Standard image processing procedures, including dark subtraction and flattening, were applied to each

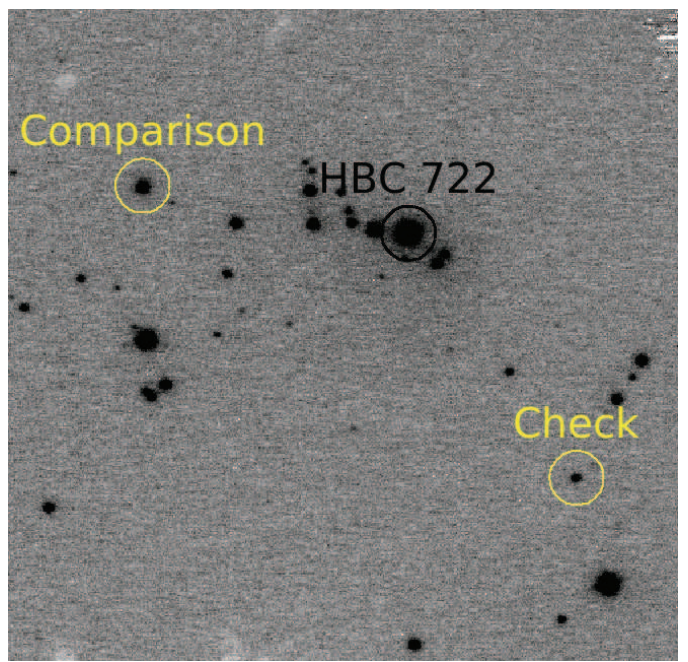

Fig. 1.- An example of a KASINICS $J$ band image showing the HBC 722 field. The target, comparison and check stars are marked with circles. North is upward and East is to the left.

object image. The influence of the background sky was also removed by subtracting the sky background image after masking out the stars. 


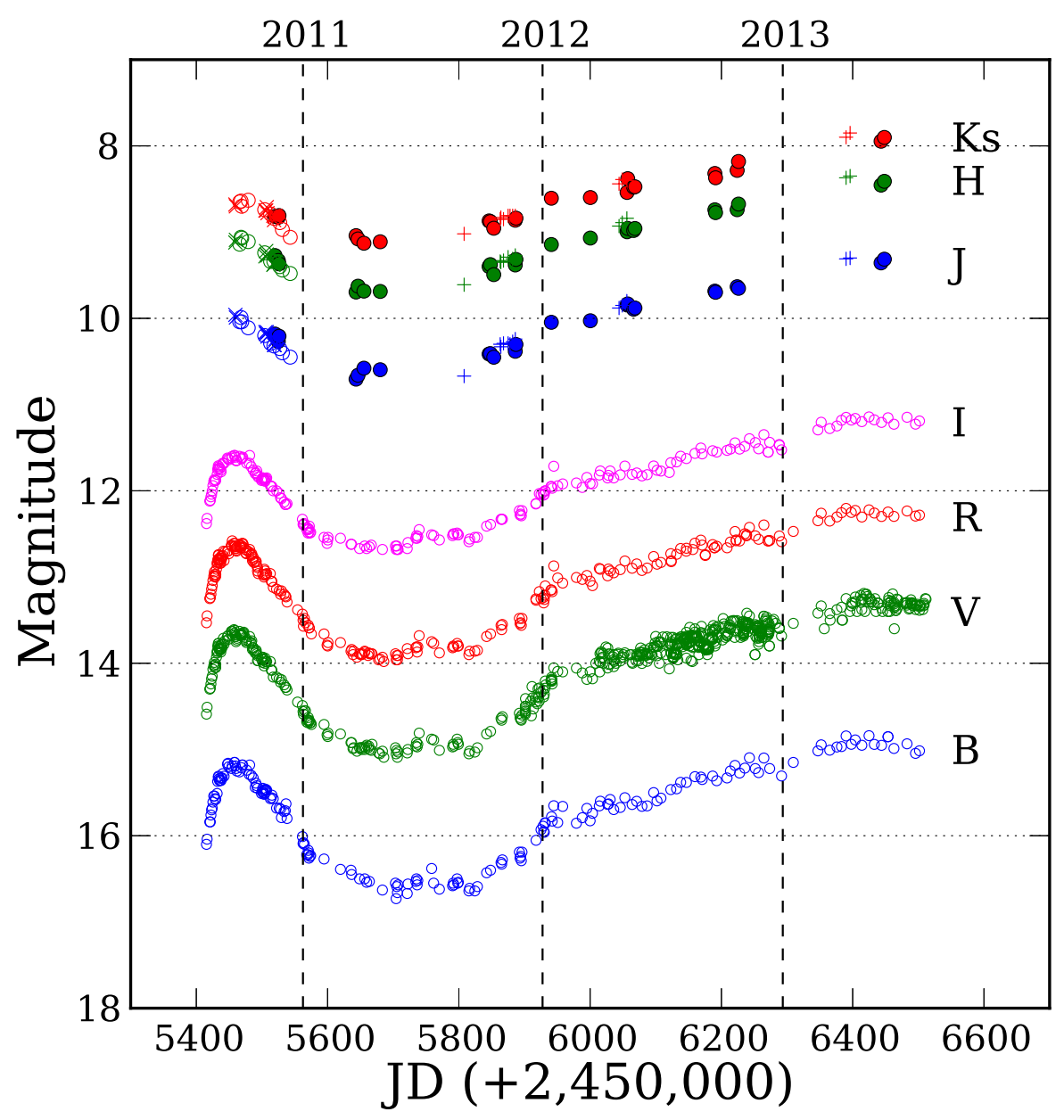

Fig. 2.- $J H K_{s}$ and $B V R I$ light curves of HBC 722. Filled dots from the $J H K_{s}$ bands are from this work, open circles are from (Miller et al. 2011), crosses are from (Kóspál et al. 2011), and plus signs are from (Antoniucci et al. 2013). The $B V R I$ data are from (Semkov et al. 2012) and the AAVSO data archive.

\subsection{Photometry}

To study both the long and short term variabilities on its light curves, we carried out the photometry on two image sets. In order to examine the long term variability, we median-combined all the images obtained in a night, to reduce the fluctuation of each image and enhance the signal - to - noise ratio of the photometry. DAOPHOT/IRAF* was used to perform aperture photometry on the combined image sets. The radius of the aperture was set to 1.5 times the FWHM of each image, after taking into account the seeing condition of each night.

For the short term variability study, we performed

*IRAF is distributed by the National Optical Astronomy Observatory, which is operated by the Association of Universities for Research in Astronomy (AURA) under cooperative agreement with the National Science Foundation. aperture photometry on all the individual images as well. Due to the sheer number of the individual images, the Source Extractor (Bertin \& Arnouts 1996) was used for the aperture photometry. To check the consistency of photometry results between DAOPHOT/IRAF and the Source Extractor, we carried out aperture photometry with DAOPHOT/IRAF on individual images for a night with the same aperture size. The comparison of two photometry results showed that the magnitude difference between the two codes is smaller than 0.02 mag. throughout the night. The radius of the aperture was fixed to $3.9^{\prime \prime}$ for all the individual images. HBC 722 developed a large nebulosity after the outburst (Miller et al. 2011), so our aperture might not have been large enough to measure all the flux. However, the target is located in a crowded region: hence, we decided to use a rather small aperture so that the photometry was not contaminated with neighbouring sources. 


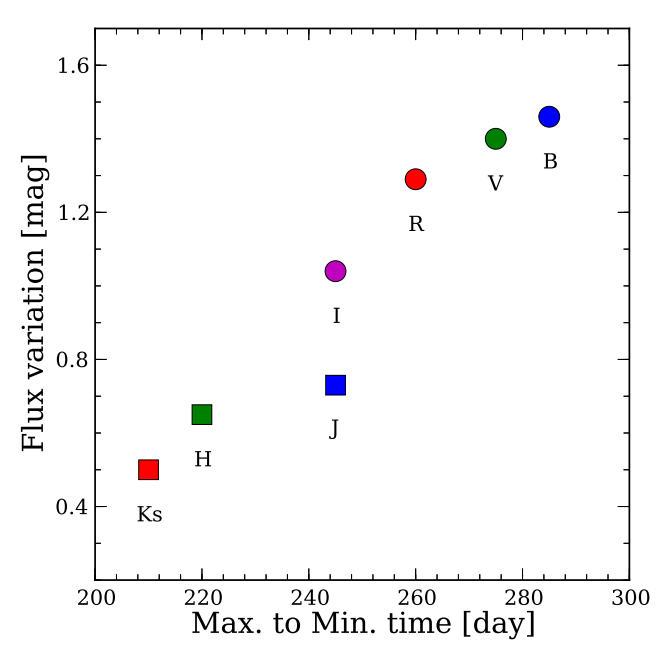

Fig. 3. - Correlation between fading period and flux variation. This strong correlation is due to the diffrential rotation and the radial density distribution of the disk.

$\mathrm{LkH} \alpha 189$ was used as the comparison star to derive the light curve of HBC 722 . Although this is an emission star, it is reported not to be a variable in the near-infrared region, based on the variability-checking result of the WISE data archive (Cutri et al. 2012). The KASINICS HBC 722 field includes one of the comparison stars used by Semkov \& Peneva (2010) (marked as a check star in Fig. 1). However, this star turned out to be faint in the near-infrared band therefore the corresponding light curve produced a much larger scatter. We used this star as a check star for the comparison star. The photometry was calibrated against the 2MASS point source catalog and compared with previous measurements.

\section{RESULTS AND DISCUSSION}

\subsection{Near-infrared Light Curve}

The $J, H$, and $K_{s}$ band near-infrared light curves of HBC 722 taken during 2.5 years (November 2010 - June 2013) are plotted in Fig. 2. We have also shown the $B, V, R$, and $I$ band optical light curves on the same figure, for comparison. In Fig. $2, J H K_{s}$ data (filled circles) represent the KASINICS observations from the present study, open circles are data from the $1.3 \mathrm{~m}$ Peters Automated Infrared Imaging Telescope (PAIRITEL) at Mt. Hopkins, AZ (Miller et al. 2011), crosses represent observations from the $1.52 \mathrm{~m}$ Telescopio Carlos Sanchez (TCS) of the Teide Observatory in the Canary Islands, Spain (Kóspál et al. 2011), and the plus signs are data from the $1 \mathrm{~m}$ IR telescope at Campo Imperatore, Italy (Antoniucci et al. 2013). Our nearinfrared observational results are consistent with other studies. The $B V R I$ data in Fig. 2 are from Semkov et al. (2012) and AAVSO (American Association of Vari-

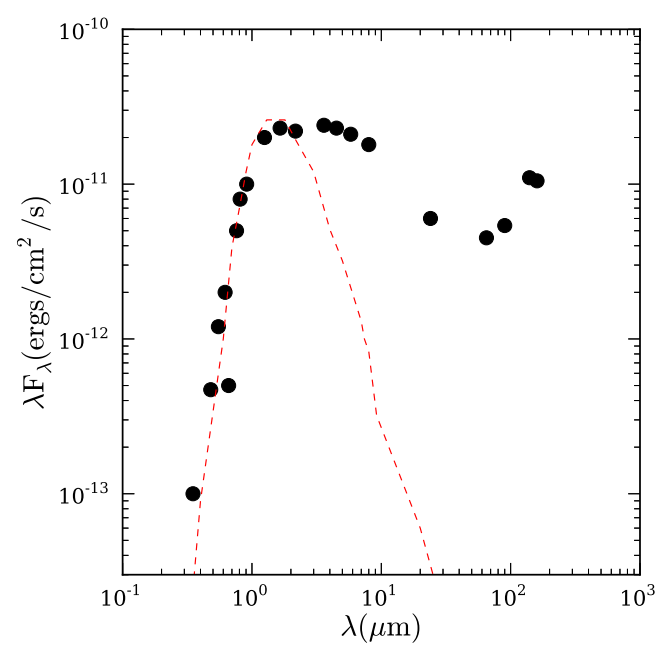

Fig. 4. - Spectral energy distribution of HBC 722 before the outburst. Optical photometry is from the SDSS, infrared data from 2MASS, Spitzer, AKARI, and WISE observations. The dashed line shows the photospheric model SED, from Robitaille et al. (2006, 2007).

able Star Observers) data archive.

The optical flux of HBC 722 declined slowly, after reaching its maximum brightness in October 2010 (Semkov et al. 2010), and darkened by 1.45 magnitudes (V-band) in the Spring/early Summer of 2011. There are no near-infrared data before the optical maximum brightness, since near-infrared observations started 5 months after the outburst. The near-infrared light curves reveal the same brightness variation trend seen in the optical light curve.

The optical and near-infrared curves reveal a second peak, seen around June 2013. Follow up observations will show the peak more clearly, which appears to be even brighter than the initial outburst peak in nearinfrared wavelengths. This pattern shows the same trend of light-curves in the optical bands. Hence, the disk component which emits the near-infrared excess flux is physically coherent with both the disk and stellar components which emit optical light.

Green et al. (2013) detected 5.3 and 1.3 day periods of $10^{-2}$ mag. amplitude scale. Many small amplitude variations are seen in the nightly light curve in KASINICS data, but we do not find the short period variability of HBC 722. One of the possible reasons is the scatter of $\sim 0.05 \mathrm{mag}$. in our light curve.

\subsection{Differential Rotation and Density Distri- bution of the Disk}

We have examined the fading period - from the maximum to the minimum brightness after the outburst - and the amplitude of the flux variation during this period by fitting a polynomial to the observed light 


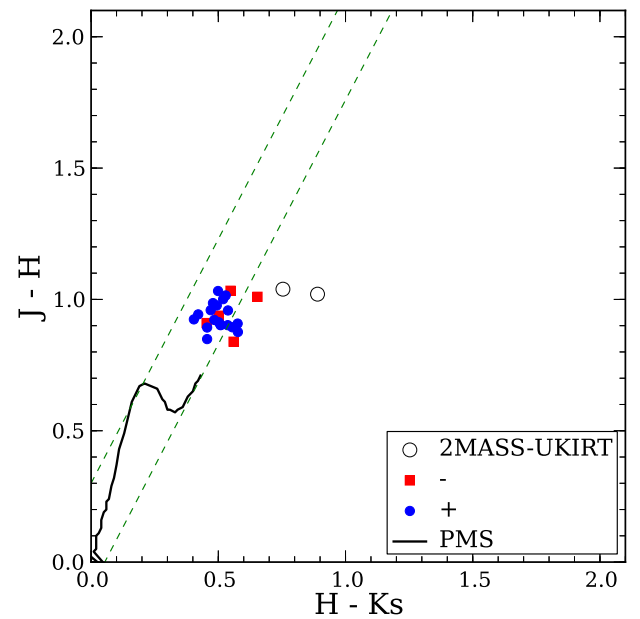

Fig. 5.- Near-infrared color-color diagram. Filled circles and squares are KASINICS data and open circles are data from 2MASS and UKIRT observations. The solid line is the pre-main-sequence track (Koornneef 1983) and the reddening path is indicated with dashed lines (Cardelli et al. 1989). Filled circles are at the brightening phase and filled squares are at the fading phase.

curves (Fig. 3). The amplitude of the flux variations in each band exhibit systematic differences, and there is a strong correlation between the fading period and the flux variation. This correlation between period and flux is due to the differential rotation and radial density distribution of the disk.

In the accretion disk model, the differentially rotating disk is expected and spectra at longer wavelengths are produced by the slowly rotating outer disk. Observations of FU Ori have shown a smaller rotation velocity at near-infrared wavelengths than that observed in optical wavelengths (Zhu et al. 2009), i.e., the widths of the spectral lines at shorter wavelengths are broader than those at longer wavelengths. This is consistent with a Keplerian rotation. According to $\mathrm{Zhu}$ et al. (2009), the spectral line width in the B-band is about two times bigger than that in the $K_{s}$ band.

If the disk does not have any motion or turbulence, the accretion speed will be the same everywhere in the disk. Instead if there is differential rotation in the disk, the velocity difference of two particles nearby the inner disk is larger than that in the outer disk $\left(\Delta v_{\text {inner }}>\Delta v_{\text {outer }}\right)$ because the rotational velocity of the inner disk is larger than that of the outer disk $\left(v_{\text {inner }}>v_{\text {outer }}\right)$. The higher velocity difference between particles acts as a higher disturbing force to the particle accretion. Therefore, the re-accretion time of the inner disk which emits optical light is longer than that of the outer disk, due to this Keplerian rotation. The fading periods are $\sim 285$ days in the B band (inner disk) and $\sim 210$ days in the $K_{s}$ band (outer disk), respectively.

The flux variations in Fig. 3 are related to the amount of re-accretion material. A first reason for the flux variations is the radial density distribution of dust particles in the disk. The density structure of the protostellar disk seems to be a power-law distribution of the dust (Yamashita et al. 1990). It takes much more time for re-accretion in the inner disk, and the amount of re-accretion material in the inner disk ( $\mathrm{B}$ band) is also longer than that in the outer disk $\left(K_{s}\right.$ band). The flux variation difference between the $\mathrm{B}$ and $K_{s}$ band was estimated as $\sim 1 \mathrm{mag}$.

\subsection{Spectral Energy Distribution}

The pre-outburst spectral energy distribution (SED) of HBC 722 is shown in Fig. 4. We compiled a preoutburst SED using data from the SDSS database, the 2MASS Point Source Catalog, the AKARI/IRAC midinfrared all-sky survey (Ishihara et al. 2010), Spitzer data (Covey et al. 2011), and WISE data. The SDSS and 2MASS data were obtained between 2000 and 2003, while Spitzer data are from 2009 and AKARI from 2010. WISE data are collected just before the outburst.

The pre-outburst SED of HBC 722 is consistent with that of a Class II YSO, also known as a T Tauri star, with the IR-excess in the far-infrared region. It is in accordance with the claim of Miller et al. (2011) that HBC 722 is a Class II object where the central star is a K7-type star. The far-infrared emissions are due to disk evolution and circumstellar material accretion. This means that dust particles are highly accumulated in the disk just before the outburst. We can interpret the farinfrared excess as an indication of FUors phenomena before the outburst.

\subsection{Near-infrared Color-color Diagram}

The location of proto-stars in the near-infrared color-color diagram reveals reddening and the physical state of the FUors such as the presence of an envelope, a disk and an emission source itself. Open circles in Fig. 5 are near-infrared colors of the 2MASS and UKIRT observations before the outburst. The HBC 722 locations in the color-color diagram are consistent with the lassical T Tauri star locus. Dashed lines show the direction of the interstellar reddening vectors (Straizys et al. 1989; Cardelli et al. 1989).

In Fig. 5, the reasons for the reddening of the nearinfrared color of HBC 722 before the outburst may be 1) circumstellar reddening by an envelope and/or 2) interstellar reddening by interstellar dust. Near-infrared colors keep getting bluer after the outburst. There is no color difference between the brightening phase and the fading phase after outburst. We suspect that it might be because most of the circumstellar material was blown out by the shock during the initial outburst. 


\section{SUMMARY}

Our near-infrared observational results of HBC 722 represent the very early evolution of the disk and circumstellar envelope on the FUors outburst. The outburst of a FUors is connected with an episode of increased mass accretion rate from the disk. Homogeneous near-infrared imaging observations of a FUors HBC 722 for 2.5 years were carried out. Near-infrared light curves of HBC 722 show that the object is getting brighter, and is still in an active state of outburst.

The near-infrared and optical light curves similarly reveal the second peak. Thus, it is clear that the inner disk optical light and outer disk near-infrared light sources are physically connected. The differential rotation of the disk is shown by the time delay of the fading period in our observations. The re-accretion rate and period after the maximum brightness were slightly changed by the differential rotation.

The progenitor of HBC 722 is a Class II YSO and deeply embedded in the circumstellar envelope. The infrared excess made by the circumstellar envelope disappeared right after the outburst. This means that circumstellar dust is a major cause of reddening in HBC 722 .

Multi-band observations of more FUors are required to understand the general nature of the differential rotation in disks and their evolution. High-resolution infrared spectroscopy will enable the study of the outer cool disk component and of the accretion.

\section{ACKNOWLEDGMENTS}

We acknowledge with thanks the variable star observations from the AAVSO International Database contributed by observers worldwide and used in this research. This research has made use of the Aladin interactive sky atlas, operated at CDS, Strasbourg, France. This research was partly supported by the Basic Science Research Program through the National Research Foundation of Korea (NRF) funded by the Ministry of Education, Science and Technology (No. 2011-0026126, No. 2012R1A1A2006759). We would like to thank the anonymous referee for the helpful comments.

\section{REFERENCES}

Antoniucci, S., Lorenzetti, D., Nisini, B., \& Giannini, T. 2013, Continuous Brightening of the Eruptive Variable V2493 Cyg (HBC 722), ATel, 4712, 1

Bertin, E., \& Arnouts, S. 1996, SExtractor: Software for Source Extraction, A\&AS, 117, 393

Cardelli, J. A., Clayton, G. C., \& Mathis, J. S. 1989, The Relationship Between Infrared, Optical, and Ultraviolet Extinction, ApJ, 345, 245

Covey, K. R., Hillenbrand, L. A., Miller, A. A., et al. 2011, PTF10nvg: An Outbursting Class I Protostar in the Pelican/North American Nebula, AJ, 141, 40
Croswell, K., Hartmann, L., \& Avrett, E. H. 1987, Mass Loss from FU Orionis Objects, ApJ, 312, 227

Cutri, R. M., et al. 2012, WISE All-Sky Data Release, VizieR On-Line Data Catalog, 2311, 0

Green, J. D., Evans, N. J. II., Kóspál, Á., et al. 2011, Disentangling the Environment of the FU Orionis Candidate HBC 722 with Herschel, ApJL, 731, 25

Green, J. D., Robertson, P., Baek, G., et al. 2013, An Analysis of the Environments of FU Orionis Objects with Herschel, ApJ, 764, 22

Hartmann, L., \& Kenyon, S. J. 1996, The FU Orionis Phenomenon, ARA\&A, 34, 207

Herbig, G. H. 1966, On the Interpretation of FU Orionis, VA, 8, 109

Herbig, G. H. 1977, Eruptive Phenomena in Early Stellar Evolution, ApJ, 217, 693

Ishihara, D., Onaka, T., Kataza, H., et al. 2010, The AKARI/IRC Mid-Infrared All-Sky Survey, A\&A, $514, \mathrm{~A} 1$

Kolotilov, E. A., \& Petrov, P. P. 1983, Studies of the FU Orionis Stars. I - Is V1515 Cygni Starting to Fade?, PAZh, 9, 171

Kolotilov, E. A., \& Petrov, P. P. 1985, Studies of the FU Orionis Stars. III - Photometry of FU Orionis, 1978-1985, PAZh, 11, 846

Koornneef, J. 1983, Near-Infrared Photometry. II - Intrinsic Colours and the Absolute Calibration from One to Five Micron, A\&A, 128, 84

Kóspál, Á., Ábrahám, P., Acosta-Pulido, J. A., et al. 2011, The Outburst and Nature of Two Young Eruptive Stars in the North America/Pelican Nebula Complex, A\&A, 527, A133

Lee, J.-E., Kang, W., Lee, S.-G., et al. 2011, High Resolution Optical Spectra of HBC 722 After Outburst, JKAS, 44, 67

Miller, A. A., Hillenbrand, L. A., Covey, K. R., et al. 2011, Evidence for an FU Orionis-Like Outburst from a Classical T Tauri Star, ApJ, 730, 80

Moon, B., Jin, H., Yuk, I.-S., et al. 2008, KASINICS: Near Infrared Camera System for the BOAO $1.8 \mathrm{~m}$ Telescope, PASJ, 60, 849

Robitaille, T. P., Whitney, B. A., Indebetouw, R., Wood, K., \& Denzmre, P. 2006, Interpreting Spectral Energy Distributions from Young Stellar Objects. I. A Grid of 200,000 YSO Model SEDs, ApJS, 167,256

Robitaille, T. P., Whitney, B. A., Indebetouw, R., \& Wood, K. 2007, Interpreting Spectral Energy Distributions from Young Stellar Objects. II. Fitting Observed SEDs Using a Large Grid of Precomputed Models, ApJS, 169, 328

Semkov, E. H., Peneva, S. P., Munari, U., Milani, A., \& Valisa, P. 2010, The Large Amplitude Outburst of the Young Star HBC 722 in NGC 7000/IC 5070, a New FU Orionis Candidate, A\&A, 523, L3 
Semkov, E., \& Peneva, S. 2010, A Possible New FUor Star in NGC 7000, ATel, 2801, 1

Semkov, E. H., Peneva, S. P., Munari, U., et al. 2012, Optical Photometric and Spectral Study of the New FU Orionis Object V2493 Cygni (HBC 722), A\&A, 542, A43

Straizys, V., Meistas, E., Vansevicius, V., \& Goldberg, E. P. 1989, Interstellar Extinction in the Area of the North America and Pelican Nebulae Complex, A\&A, 222, 82

Weintraub, D. A., Sandell, G., \& Duncan, W. D. 1991, Are FU Orionis Stars Younger than T Tauri Stars? Submillimeter Constraints on Circumstellar Disks, ApJ, 382, 270

Yamashita, T., Sato, S., Kaifu, N., \& Hayashi, S. S. 1990, The Density Structure of the Protostellar Disk - A Power-Law Distribution of the Dust around GGD 27 IRS, ApJ, 365, 615

Zhu, Z., Espaillat, C., Hinkle, K., et al. 2009, The Differential Rotation of FU Ori, ApJL, 694, 64 